\title{
Abrupt changes, thresholds, and tipping points in Earth's history and future implications
}

\author{
Ed Brook ${ }^{1}$ and Victor Brovkin ${ }^{2}$ \\ AIMES-PAGES joint workshop, Hamburg, Germany, 14-16 November 2018
}

\begin{abstract}
There is increasing realization and concern that human modification of the Earth system runs the risk of inducing abrupt transitions in climate, ocean circulation, the cryosphere, ecosystems, and society (Turney et al. 2016). Our ability to predict when and where such transitions, so-called "tipping points", might happen is limited. While abrupt climate change has long been identified in ice-core records (Johnsen et al. 1992) and other archives, skillfully modeling abrupt change has largely been limited to simple models (Valdes 2011). Recently, a multi-model assessment revealed abrupt events in state-ofthe-art models, suggesting the possibility of predicting the likelihood of such events (Drijfhout et al. 2015)
\end{abstract}

Advancing our understanding of the full range of possible abrupt climate, environmental, and societal changes through the continued assessment and collection of paleo data and improved multi-model experiments will help us to assess future risks. However, how to best use the paleo records in this regard is not well established. The AIMES-PAGES workshop on abrupt changes, thresholds, and tipping points in the Earth system was organized around this question. Both PAGES and AIMES (Analysis, Integration and Modelling of the Earth System; aimesproject.org) are Future Earth Global Research Projects. Within PAGES, the topic of "Thresholds, tipping points and multiple equilibria in the Earth system" is an established integrative activity. Within AIMES, the assessment of tipping points is identified as one of the four major AIMES projects. The workshop was an outgrowth of a splinter group meeting at the 2018 EGU General Assembly, organized by PAGES, that explored community interest in this topic.

Workshop attendees were drawn from a diversity of subdisciplines in paleoenviromen tal modeling, data collection, and socialenvironmental systems. Following a series of introductory talks from the perspective of different components of the Earth system (terrestrial and marine environments, the cryosphere, the atmosphere, and humansocial systems), breakout groups spent a full day in focused discussion organized around the physical climate system, ecosystems, biogeochemical cycles, and social systems. The primary task of the groups was to evalu ate the potential for significant progress in using paleoclimate data to predict future tipping points and abrupt change.

All of the breakout groups reported that significant work is needed to fully take advantage of the paleo record for evaluating the potential for crossing critical thresholds in the near future. Topics emphasized include cryospheric change and its cascading impacts (including sea-level change and permafrost thaw), hydroclimate instabilities (including changes in fire, wetlands, and rainfall), oceanic changes (including hypoxia and rapid changes in ocean circulation), and the interaction of human societies with these changes. Early detection of abrupt transitions is another area of considerable interest that requires further attention.

The groups agreed that it is vital to include the paleo perspective in evaluating future tipping points and related changes, given model uncertainties and the short timescale of direct observations. A synthesis paper describing the future challenges and opportunities in this area is in preparation, and planning for follow-up research and workshops is underway.

\section{AFFILIATIONS}

'Oregon State University, Corvallis, USA

${ }^{2}$ Max Planck Institute for Meteorology, Hamburg, Germany

\section{CONTACT}

Ed Brook: brooke@geo.oregonstate.edu

REFERENCES

Drijfhout S et al. (2015) Proc Natl Acad Sci 112: E5777-E5786

Johnsen SJ et al. (1992) Nature 359: 311-313

Lenton TM et al. (2008) Proc Natl Acad Sci 105: 1786-1793

Turney CSM et al. (2016) PAGES Magazine 24: 1-52

Valdes P (2011) Nat Geosci 4: 414-416

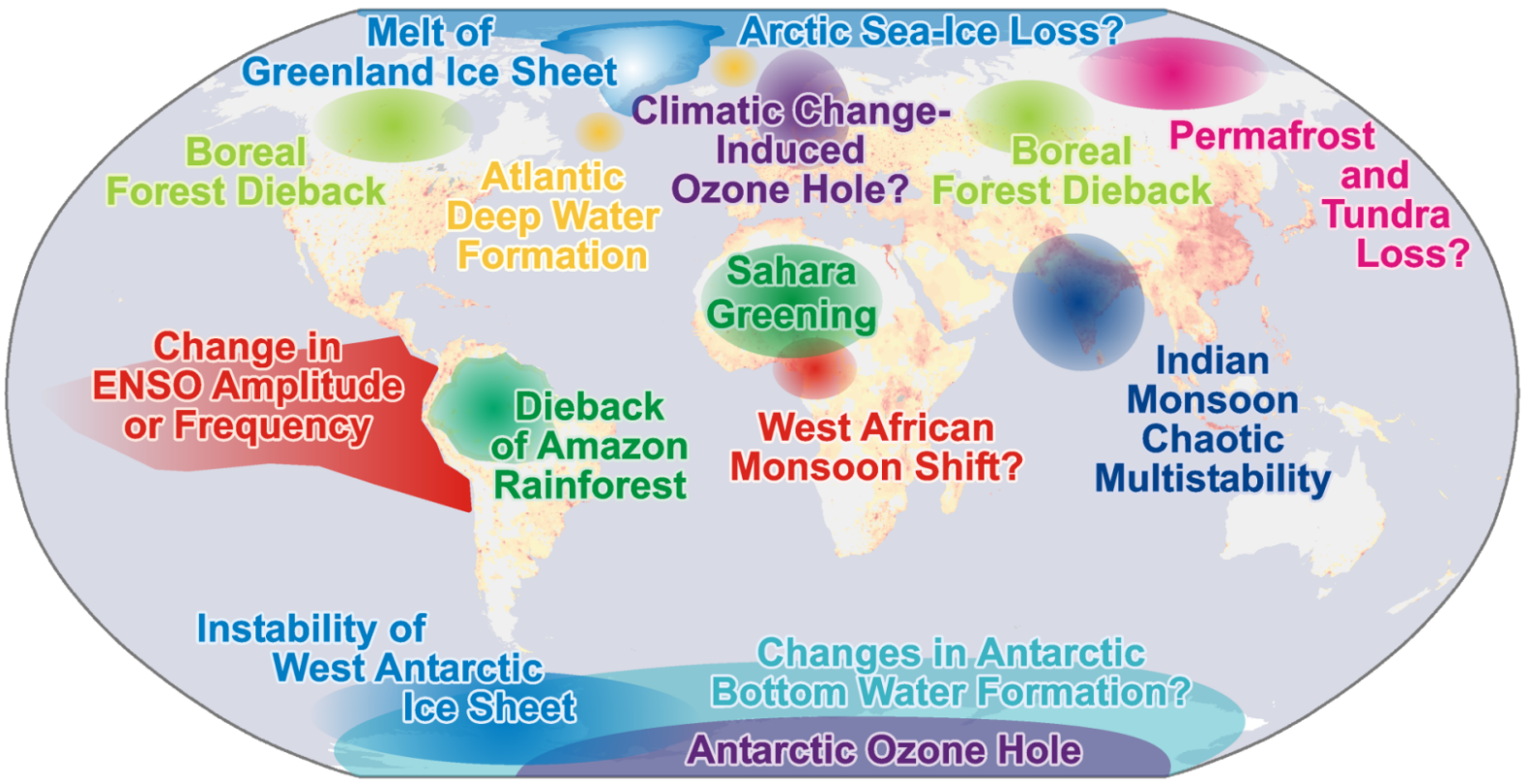

Figure 1: Climate system elements that may be susceptible to tipping point behavior, with background shading showing population density. Modified from Lenton et al. (2008); copyright 2008 National Academy of Sciences. 